\title{
Ciclos económicos, expectativas e inflación en el Brasil: análisis a partir de la curva de Phillips neokeynesiana
}

\author{
Elano Ferreira Arruda, Maria Thalita Arruda Oliveira \\ de Olivindo e Ivan Castelar
}

\section{Resumen}

En este estudio se analiza la dinámica reciente de la inflación brasileña considerando distintas hipótesis de expectativas para observar la manera en que un posible comportamiento discrecional de la autoridad monetaria puede interferir en las expectativas con miras al futuro de los agentes y la forma en que esa interferencia puede afectar la respuesta de la inflación a su componente inercial y a las oscilaciones en los ciclos económicos en el marco de la curva de Phillips neokeynesiana. Para ello se realizan estimaciones de dicha curva y de su versión híbrida con el método generalizado de momentos (MGM) consistente en presencia de heterocedasticidad y autocorrelación. Los resultados sugieren que, en una hipótesis de menor previsibilidad de los agentes, la inflación será más sensible a las oscilaciones en los ciclos económicos cuanto mayor sea su componente inercial.

\section{Palabras clave}

Condiciones económicas, ciclos económicos, inflación, análisis económico, modelos econométricos, Brasil

\section{Clasificación JEL}

E30, E31, E32

\section{Autores}

Elano Ferreira Arruda es Profesor Adjunto del Programa de Posgrado en Economía (CAEN/UFC) y Maestría Académica en Economía Rural (MAER/UFC) de la Universidad Federal de Ceará, Brasil. Correo electrónico: elano@ufc.br.

Maria Thalita Arruda Oliveira de Olivindo es alumna de Doctorado del Programa de Posgrado en Economía de la Universidad Federal de Ceará (CAEN/UFC), Brasil. Correo electrónico: thalitaoliveira.sobral@gmail.com.

Luiz Ivan de Melo Castelar es Profesor Titular del Programa de Posgrado en Economía (CAEN/UFC) de la Universidad Federal de Ceará, Brasil. Correo electrónico: lume1250@yahoo.com.br. 


\section{Introducción}

La curva de Phillips, inicialmente representada por la compensación entre la inflación de los salarios y el desempleo, fue objeto de importantes transformaciones a lo largo de las últimas décadas. Esas modificaciones obedecieron tanto a la evolución de la teoría macroeconómica como al surgimiento de nuevos hechos estilizados sobre la relación entre los ciclos económicos, las expectativas y la inflación.

La modificación más reciente, basada en las formulaciones de Taylor (1980) y Calvo (1983), se conoce como curva de Phillips neokeynesiana. Esta se deriva a partir de un modelo microfundamentado de expectativas racionales, que prevé una relación entre la inflación a corto plazo y una medida del costo marginal de las empresas y destaca la importancia de las expectativas con miras al futuro. Asimismo, Galí y Gertler (1999) proponen la denominada curva de Phillips neokeynesiana híbrida, en la cual se evalúa también la participación del componente inercial, o basado en el pasado (backwardlooking), de la inflación.

En la actualidad, la curva de Phillips neokeynesiana y su versión híbrida se encuentran en el centro del debate sobre la conducción de la política monetaria y la relación entre los ciclos económicos, las expectativas y la inflación ${ }^{1}$. Esto es relevante porque una autoridad monetaria creíble puede proyectar una política de desinflación sin costos si la inflación es un proceso puramente prospectivo, mientras que, en presencia de un componente retrospectivo, el costo de la referida política en términos de actividad económica podría ser mayor.

La literatura internacional revela cierta polarización en los estudios sobre el tema, pues algunos autores muestran que la curva de Phillips neokeynesiana es un mecanismo robusto para explicar la dinámica inflacionaria (Galí y Gertler, 1999; Galí, Gertler y López-Salido, 2001), mientras que otros cuestionan su relevancia empírica (Rudd y Whelan, 2005). En el caso del Brasil, en diversos trabajos se presentan evidencias de la importancia de la curva de Philips para analizar el comportamiento de la inflación, a pesar de que los resultados son bastante sensibles a los métodos de estimación y a las variables representativas empleadas en el modelo (Mendonça, Sachsida y Medrano, 2012; y Sachsida, 2013).

Las principales evidencias sobre la economía brasileña muestran la relevancia estadística de los componentes de expectativas con miras al futuro y basadas en el pasado en el comportamiento de la inflación y la dificultad de la variable ciclo económico - medida por la brecha del productopara captar el efecto de los cambios en la actividad económica en la dinámica inflacionaria (Areosa y Medeiros, 2007; Sachsida, Ribeiro y Dos Santos, 2009; Arruda, Ferreira y Castelar, 2011)².

A pesar del amplio debate sobre la idoneidad de la curva de Phillips neokeynesiana, poco se ha discutido sobre la manera en que la dinámica reciente de la inflación brasileña responde a su componente inercial y a los ciclos de la actividad económica considerando diferentes niveles de previsibilidad de los agentes, o expectativas con miras al futuro, en la curva de Phillips neokeynesiana y en su versión híbrida, visto que autores como Mendonça (2002 y 2004), Sicsú (2002) y Mendonça y Santos (2006) muestran que la credibilidad de la autoridad monetaria afecta el poder predictivo de los agentes económicos y que gran parte de la inercia de la inflación se debe a la pérdida de reputación del gobierno en virtud del incumplimiento de las reglas previamente firmadas con los agentes.

Por tanto, en el presente estudio se procura examinar el comportamiento reciente de la inflación brasileña considerando distintas hipótesis de expectativas en la curva de Phillips neokeynesiana y la curva de Phillips neokeynesiana híbrida. En otras palabras, se desea responder a las siguientes preguntas: ¿cómo se comportan los coeficientes estimados de la curva de Phillips neokeynesiana

\footnotetext{
1 Ciclos económicos en el sentido de costo marginal de las empresas.

2 Véase una buena revisión de las principales evidencias en Mendonça, Sachsida y Medrano (2012) y Sachsida (2013).
} 
y de su versión híbrida en hipótesis de expectativas con miras al futuro diferenciadas?, ¿la inflación sería menos sensible a las oscilaciones cíclicas del producto y a su componente inercial si los agentes económicos realizaran una previsión perfecta?, ¿aumentan los costos de una política de desinflación - en términos de reducción de la actividad económica - en una hipótesis de menor previsibilidad de los agentes? Visto que autores como Simonsen (1970 y 1985), Cabello (2014) y Carvalho (2014) advierten que la permanencia o el aumento de la indexación tienen repercusiones importantes en la realimentación inflacionaria, ¿influyó el posible aumento reciente del grado de indexación de la economía brasileña en el impacto de la inflación inercial?

Para ello se realizan estimaciones de la curva de Phillips neokeynesiana y de su versión híbrida para la economía brasileña basadas en el método generalizado de momentos (MGM) consistente en presencia de heterocedasticidad y autocorrelación y datos mensuales del período comprendido entre enero de 2002 y diciembre de $2012^{3}$. Se utilizan la brecha del producto, la brecha de desempleo y el costo marginal real de las empresas como variables de los ciclos económicos, la inflación medida por el Índice Nacional de Precios al Consumidor Amplio (IPCA) rezagado como medida de expectativas basadas en el pasado, el IPCA adelantado como medida de expectativas con miras al futuro bajo previsión perfecta y, por último, la media de las expectativas de inflación en todos los días del mes para el mes siguiente del informe Focus del Banco Central del Brasil como medida de expectativa con miras al futuro en una hipótesis de menor previsibilidad de los agentes económicos ${ }^{4}$.

Así, la principal contribución de este estudio reside en la evaluación del comportamiento de las respuestas de la inflación brasileña a las oscilaciones en los ciclos económicos y en su componente inercial en distintas hipótesis de expectativas con miras al futuro, además de probar la brecha de desempleo como medida del ciclo económico y utilizar una medida de costo marginal de las empresas conforme Galí y Gertler (1999) 5 . Cabe destacar que la medida de costo marginal empleada en este estudio todavía no se ha utilizado en aplicaciones para el Brasil6.

El presente estudio se divide en siete secciones, incluida esta introducción. Mientras que en la segunda se describe la evolución de la inflación brasileña en los últimos años, en la tercera se presentan algunos aspectos teóricos sobre la curva de Phillips neokeynesiana y la curva de Phillips neokeynesiana hibrida y sobre las relaciones entre los ciclos económicos, las expectativas y la inflación en distintas hipótesis de previsibilidad de los agentes. En la cuarta sección se describen y analizan los datos, mientras que en la quinta se presenta la estrategia econométrica. En la sexta sección se procede al análisis y la discusión de los resultados y, en la séptima y última, se formulan las consideraciones finales del estudio.

\section{Evolución reciente de la inflación en el Brasil}

En el intento de contener la hiperinflación que devastó la economía brasileña desde los años ochenta hasta comienzos de la década de 1990, se implementaron, sin éxito, muchos planes de estabilización. Solo con el Plan Real, inicialmente basado en el trípode constituido por un ancla cambiaria, un fuerte ajuste fiscal y una base monetaria rígida, la inflación disminuyó considerablemente entre 1995 y 1998. En ese período se redujo el grado de indexación y, en consecuencia, la inercia inflacionaria que erosionaba la moneda brasileira retrocedió.

3 Este período se eligió debido a la disponibilidad de datos para la variable de costo marginal de las empresas utilizadas.

4 El costo marginal real de las empresas se mide en términos de razón entre la masa salarial y el producto interno bruto (PIB).

5 La brecha de desempleo como medida del ciclo económico es una variable extraída de la relación de Okun, que prevé una relación inversa entre los ciclos del producto y de desempleo.

6 Normalmente, se utilizan la brecha del producto, la brecha de desempleo y el uso de la capacidad instalada de la industria, entre otros. Sin embargo, ninguno de esos indicadores se refiere efectivamente a una medida del costo marginal de las empresas. 
Debido a la marcada valorización de la moneda nacional, el país acumuló un elevado déficit en cuenta corriente y, por lo tanto, se volvió dependiente del ingreso de capital internacional y más vulnerable a choques externos (Giambiagi y otros, 2011). Así, después de tres ataques especulativos contra el real, a saber, la crisis de México (1995), la crisis de Asia (1997) y la moratoria de Rusia (1998), el incremento de la tasa de interés ya no bastaba para impedir la fuga de capital y la continua pérdida de reservas forzó la devaluación del tipo de cambio en 1999.

Después de la eliminación del ancla cambiaria, se adoptó el sistema de metas de inflación como un instrumento para evitar que la indexación volviera a instalarse en la economía brasileña. De ese modo, con el ancla nominal definida, el Banco Central fijó las metas para 1999 (8\%), 2000 (6\%) y 2001 (4\%), con un intervalo de tolerancia de dos puntos porcentuales. Al contrario de lo esperado por quienes temían el regreso de la inflación, la devaluación del tipo de cambio no tuvo efectos inflacionarios y el IPCA se mantuvo dentro del intervalo previsto en 1999 y $2000^{7}$. A partir de entonces, el nuevo trípode estaría representado por metas de inflación, tipo de cambio flotante y responsabilidad fiscal (superávits primarios). En el gráfico 1 se presenta la evolución de la inflación en ese período.

\section{Gráfico 1}

Brasil: evolución de la inflación (Índice Nacional de Precios al Consumidor Amplio (IPCA)) después del establecimiento del sistema de metas, 2001-2015 (En porcentajes)

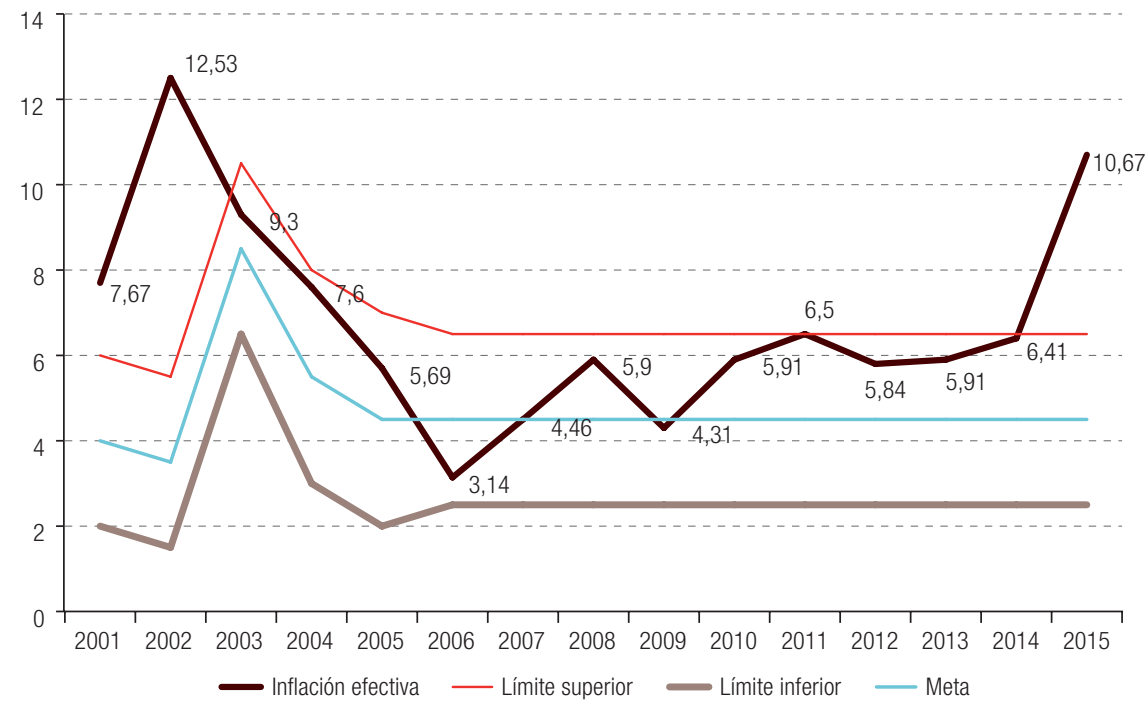

Fuente: Elaboración propia.

La situación se agravó con la campaña electoral de 2002, debido a la incertidumbre sobre lo que ocurriría con la política económica a partir de 2003. El centro de la meta del IPCA fijado para 2002 en el año 2000 era del 3,5\%. En enero de 2002 la economía brasileña presentaba una inflación del 7,62\% en el acumulado de los últimos 12 meses, que aumentó gradualmente hasta llegar al 7,93\% en septiembre de ese año. A partir de octubre, cuando Luiz Inácio Lula da Silva ganó las elecciones, la inflación aumentó considerablemente, pasando del 8,45\% ese mes al 12,53\% al finalizar el año.

Es en esa coyuntura que, en enero de 2003, el Banco Central fija una nueva meta de inflación para ese año, pasando del 3,25\% al 8\%. Cuando el nuevo gobierno asumió una conducta en línea con la política monetaria anterior, que indicaba que las prioridades eran el control de la inflación y

\footnotetext{
7 El Índice Nacional de Precios al Consumidor Amplio (IPCA) se eligió como medida de referencia para las metas debido a su característica de resultar afectado por factores estacionales y choques temporarios.
} 
la responsabilidad fiscal, comenzó a superarse la crisis de confianza y se retomó la trayectoria de reducción de la tasa de inflación, cuyo valor fue del 9,3\% en 2003.

Bresser-Pereira y Gomes da Silva (2008) destacan que, si bien en 2004 hubo una extraordinaria sustitución de ahorro externo por ahorro interno, el Banco Central mantuvo la tasa de interés elevada y, además se observó un aumento en las exportaciones. Esto se tradujo en una apreciación del tipo de cambio, que contribuyó a la reducción del IPCA y al alcance de la meta. A partir de ese momento, la inflación presentó una tendencia decreciente hasta 2006.

La trayectoria decreciente se consolidó con la fijación de la meta para 2005 en un 4,5\%. Entre 2004 y 2014, la inflación se mantuvo siempre dentro del intervalo de la meta ${ }^{8}$. Ese resultado obedeció principalmente a la apreciación del real. Sin embargo, la crisis financiera de 2007-2008 paralizó las fuentes de crédito externo y provocó una devaluación del tipo de cambio. A pesar de ello, la inflación se mantuvo por debajo de la meta.

No obstante, el IPCA alcanzó el límite máximo de la meta en 2011, a raíz de la evolución de los precios de los productos básicos - sobre todo energéticos y alimenticios - y del aumento del precio de las monedas internacionales con respecto a la moneda nacional como consecuencia de la crisis internacional de 2011. Cabe destacar también ese año la asunción de la presidencia por Dilma Rousseff y su compromiso con la nueva matriz económica, caracterizada por la expansión fiscal, el crédito abundante a intereses subsidiados y el tipo de cambio controlado. La reducción de la tasa de interés comenzó en el segundo semestre de 2011 y continuó a lo largo de 2012 e inicios de 2013, al pasar del $12,42 \%$ al $7,11 \%$.

Además, la política de reajuste del salario mínimo vinculado a la inflación aumentó el grado de indexación de la economía y, con ella, la inflación inercial. Durante ese período la inflación se mantuvo lejos del centro de la meta y, por el contrario, cada año se acercó más a su límite máximo. Incluso con la reanudación del aumento de la tasa del Sistema Especial de Liquidación y Custodia (SELIC), en 2014, el IPCA llegó al 6,41\%.

De ese modo, se abandonó el sistema de metas de inflación a favor de la expansión del crédito público a intereses subsidiados. El enfoque en el desarrollismo y la negligencia de la estabilidad monetaria y el equilibrio fiscal resultaron evidentes. De acuerdo con Mesquita (2014), para combatir la inflación se optó por las exenciones arancelarias y el control artificial de los precios sobre los cuales el poder público tiene injerencia (precios administrados). El Estado también intervino en la política cambiaria, al anunciar en agosto de 2013 un programa continuo de seguro de cambio, por medio de intervenciones diarias. Con respecto a la política fiscal, se observó un deterioro constante del resultado primario, que culminó en un déficit de 32.000 millones de reales en 2014. En ese contexto, la inflación superó el límite máximo en 2015.

\section{Revisión de la literatura}

\section{Curva de Phillips tradicional}

La versión original de la curva de Phillips muestra una relación inversa entre la inflación de los salarios y el desempleo. En su versión tradicional, la curva tiene la siguiente especificación:

$$
\pi_{t}=\alpha+\gamma u_{t}+\varepsilon_{t}
$$

donde $\pi_{t}$ es la inflación de los salarios en $t, u_{t}$ es la tasa de desempleo corriente y $\alpha$ y $\gamma$ son los parámetros en que $\gamma<0$.

8 La meta de inflación continuó fijada en un 4,5\% al año, a partir de 2006 la banda se cambió al intervalo del 2,5\%-6,5\%. 
De acuerdo con Phillips (1958), una tasa de desempleo elevada indicaría un exceso de oferta de mano de obra y, en consecuencia, habría presión para que los salarios disminuyeran. Surgía, así, la compensación entre inflación y desempleo que las economías enfrentan en la elaboración y ejecución de políticas económicas. Estas disponían del modelo IS-LM para analizar el lado de la demanda, junto con la curva de Phillips, que representaba el lado de la oferta.

Sin embargo, la creencia de que una variable nominal (inflación) afectaba variables reales (tasa de desempleo) fue bastante criticada en la segunda mitad de la década de 1960. Autores como Phelps (1967 y 1969) y Friedman (1968 y 1977) argumentaron que la formulación original de la curva procura analizar el crecimiento de los salarios nominales frente a las tasas de desempleo, lo que contradice la hipótesis de racionalidad de los agentes económicos, pues estos se preocupan por la evolución de variables reales y no simplemente de variables nominales. De acuerdo con esos autores, la curva de Phillips necesitaba un componente para captar las expectativas de inflación.

Con la reducción de la tasa de desempleo y, con ello, el aumento de la tasa de inflación, los trabajadores percibirían que la inflación era mayor que la esperada y negociarían los salarios sobre la base de esta nueva expectativa. En consecuencia, la tasa de desempleo volvería a su estado original, pues los salarios reales, que habían disminuido, volverían a su nivel anterior. Esta idea inicial de Muth (1961) se denominaría expectativas adaptativas, o basadas en el pasado, pues los agentes corrigen sus expectativas sobre la base de los errores de previsión pasados, o sea, la expectativa de inflación, $\pi_{t}^{e}$, se modelaría como una media ponderada de las tasas de inflación pasadas, atribuyéndose mayor peso a los datos más recientes. La curva de Phillips asumiría la forma

$$
\pi_{t}=\varphi \pi_{t}^{e}+\gamma\left(u_{t}-u_{n}\right)+\varepsilon_{t}
$$

donde $\pi_{t}$ es la inflación corriente, $\pi_{t}^{e}$ la expectativa de inflación para $t$, dada por una media ponderada de las tasas de inflación pasadas, $u_{t}$ la tasa de desempleo corriente y $u_{n}$ la tasa de desempleo natural.

De este modo, las expectativas de los agentes pasaron a ser fundamentales en la construcción y ejecución de las políticas económicas. Sin embargo, posteriormente se constató que, sobre la base de las expectativas adaptativas, los agentes económicos cometerían errores sistemáticos de previsión, un hecho que no resulta razonable en la realidad.

Esta constatación produjo una revolución en la teoría macroeconómica entre las décadas de 1970 y 1980, impulsada por la hipótesis de expectativas racionales, o con miras al futuro, atribuida a Lucas (1972) y Sargent (1971). Según esos autores, al definir la inflación esperada los agentes económicos tienen en cuenta toda la información a su alcance hasta el período corriente y no solo una combinación de los datos pasados. Así, $\pi_{t}^{e}$ pasa a ser una función del conjunto de datos disponibles hasta $t$, de manera que surge la necesidad de incorporar un componente con miras al futuro en la curva de Phillips.

\section{Curva de Phillips neokeynesiana}

En las últimas décadas se ha discutido ampliamente un nuevo enfoque de la curva de Phillips. Taylor (1980) y Calvo (1983) sentaron las bases para el análisis moderno de la inflación examinando la elección de los precios y los salarios en una perspectiva con miras al futuro por parte de las familias y las empresas. En esta versión, la curva se deduce como una relación entre la inflación y el costo marginal de las empresas. Esta formulación, denominada curva de Phillips neokeynesiana, parte de dos ecuaciones estructurales, a saber:

$$
p_{t}=\theta p_{t-1}+(1-\theta) p_{t}^{*}
$$




$$
p_{t}^{*}=(1-\beta \theta) \sum_{k=0}^{\infty}(\beta \theta)^{k} E_{t}\left\{c m_{t+k}^{n}\right\}
$$

En la ecuación (3), $p_{t}$ es el nivel de precios agregado y $p_{t}^{*}$ es el nivel de precios derivado de la maximización del beneficio de las empresas, ambos en logaritmo, y $\theta(0<\theta<1)$ define la fracción de empresas que no ajustan sus precios mediante la optimización de los beneficios en $t$. O sea, la ecuación (3) introduce cierta rigidez de precios, visto que solo una fracción $(1<\theta)$ de las empresas puede ajustar de manera óptima sus precios en $t$ mientras las demás mantienen el nivel de precios del período anterior.

La ecuación (4) puede derivarse formalmente a partir de la maximización del valor presente del beneficio esperado por parte de las empresas y especifica el precio óptimo elegido por las empresas como función de $\theta$, del costo marginal real, $\mathrm{cm}_{t+k}^{n}$, y de un factor de descuento $\beta$ (Calvo, 1983). O sea, en ausencia de fricciones o costos de ajuste, las empresas definirían sus precios igualando el costo marginal en cada período. Sin embargo, las empresas no cambian sus precios en todos los períodos, por eso la definición de $p_{t}^{*}=c m_{t+k}^{n}$ no es adecuada en este contexto. Entonces, los precios deben formarse a partir de la expectativa del comportamiento del costo marginal esperado de manera de maximizar el valor presente del beneficio esperado. Así, al definir la inflación en $t$ como $\pi_{t}=p_{t}-p_{t-1}$ y combinar las ecuaciones (3) y (4) se obtiene la curva de Phillips neokeynesiana expresada como:

$$
\pi_{t}=\lambda c m_{t}+\gamma_{f} E_{t}\left\{\pi_{t+1}\right\}
$$

En otras palabras, la inflación corriente será una función del costo marginal real de las empresas en $t$ y de la expectativa de inflación, que se construye con miras al futuro. Galí y Gertler (1999) muestran que existe una relación entre el costo marginal y la brecha del producto ${ }^{9}$. Esta se expresa como:

$$
c m_{t}=k x_{t}
$$

donde $k$ es la elasticidad del costo marginal real en relación con la brecha del producto. De este modo, al sustituirla en la ecuación (5), se obtiene,

$$
\pi_{t}=\lambda k x_{t}+\gamma_{f} E_{t}\left\{\pi_{t+1}\right\}
$$

En consecuencia, la inflación en $t, \pi_{t}$, se expresará como una función de la tasa de inflación esperada para el próximo período, $E_{t}\left\{\pi_{t+1}\right\}$, es decir, un término orientado al futuro, y por una medida del ciclo económico, $x_{t}$. Así, la curva de Phillips neokeynesiana puede estimarse utilizando variables del costo marginal de las empresas y de los ciclos económicos ${ }^{10}$.

\section{Curva de Phillips neokeynesiana híbrida}

A pesar de ello, la curva de Phillips neokeynesiana todavía presenta una importante laguna, pues no incluye un componente inercial o basado en el pasado en la inflación. Esto motivó el surgimiento de la versión conocida como forma híbrida de la curva de Phillips neokeynesiana (Galí y Gertler, 1999). En este contexto, $p_{t}^{*}$ asume la forma:

$$
p_{t}^{*}=(1-\omega) p_{t}^{f}+\omega p_{t}^{b}
$$

\footnotetext{
9 La desviación (en log) del producto con respecto al nivel de producto potencial. O sea, $x_{t} \equiv \hat{Y}_{t}-\hat{Y}_{t}^{n}$.

${ }^{10}$ En el presente estudio se utilizan la brecha del producto, la brecha de desempleo y el costo marginal real de las empresas.
} 
en que $p_{t}^{f}$ es el precio fijado por las empresas que usan expectativas con miras al futuro y $p_{t}^{b}$ es el precio del conjunto de empresas que usan expectativas basadas en el pasado ${ }^{11}$. El primer grupo de empresas se comporta exactamente como en el modelo descrito por Calvo (1983). Así, $p_{t}^{f}$ será:

$$
p_{t}^{f}=(1-\beta \theta) \sum_{k=0}^{\infty}(\beta \theta)^{k} E_{t}\left\{c m_{t+k}^{n}\right\}
$$

El precio de las empresas que forman sus expectativas basadas en el pasado se expresará como el nivel de precios en el último período corregido por la inflación. Formalmente,

$$
p_{t}^{b}=p_{t-1}^{*}+\pi_{t-1}
$$

Por lo tanto, al combinar las ecuaciones (3), (8), (9) y (10) se puede derivar la versión híbrida de la curva de Phillips neokeynesiana, o sea,

$$
\pi_{t}=\lambda c m_{t}+\gamma_{f} E_{t}\left\{\pi_{t+1}\right\}+\gamma_{b} \pi_{t-1}
$$

donde $\gamma_{f}$ es el parámetro del término con miras al futuro, $\gamma_{b}$ incorpora el componente de la inflación basado en el pasado y $\lambda$ incorpora la contribución del costo marginal de las empresas/los ciclos económicos. Ahora, $\gamma_{b}$ pasa a indicar el grado de persistencia inflacionaria. Cabe destacar que, si $\gamma_{f}$ es estadísticamente igual a 0 se obtiene la formulación tradicional de la curva de Phillips.

\section{Descripción y análisis de los datos}

Los datos mensuales utilizados para la estimación de la curva de Phillips neokeynesiana y de su versión híbrida abarcan de enero de 2002 a diciembre de 2012. El período de la muestra se eligió en función de la disponibilidad de datos para las variables de costo marginal real de las empresas y brecha de desempleo ${ }^{12}$. En el cuadro 1 se presenta un resumen de las variables utilizadas.

\begin{tabular}{|c|c|c|c|}
\hline Indicador & Variable & Variable representativa & Fuente \\
\hline Inflación & Inflación & $\begin{array}{l}\text { Inflación Índice Nacional de Precios } \\
\text { al Consumidor Amplio (IPCA) }\end{array}$ & $\begin{array}{l}\text { Instituto Brasileño de Geografía y Estadística } \\
\text { (IBGE)/ Sistema Nacional de Índices } \\
\text { de Precios al Consumidor (SNIPC) }\end{array}$ \\
\hline \multirow{3}{*}{ Expectativas } & \multirow{2}{*}{ Expectativa con miras al futuro } & $\begin{array}{l}\text { Inflación IPCA adelantado } \\
\text { (previsión perfecta) }\end{array}$ & IBGE/SNIPC \\
\hline & & $\begin{array}{l}\text { Previsión Focus } \\
\text { (hipótesis de incertidumbre) }\end{array}$ & Informe Focus del Banco Central del Brasil \\
\hline & Expectativa basada en el pasado & Inflación IPCA rezagado & IBGE/SNIPC \\
\hline \multirow{3}{*}{$\begin{array}{l}\text { Ciclos } \\
\text { económicos }\end{array}$} & Costo marginal real & Masa salarial/producto interno bruto (PIB) & $\begin{array}{l}\text { Construida a partir de datos del IBGE } \\
\text { y del Banco Central del Brasil }\end{array}$ \\
\hline & Ciclo del producto & Brecha del producto & $\begin{array}{l}\text { Construida a partir de datos del Banco } \\
\text { Central del Brasil y filtro de Hodrick-Prescott }\end{array}$ \\
\hline & Ciclo del desempleo & Brecha de desempleo & $\begin{array}{l}\text { Construida a partir de datos de la encuesta } \\
\text { de empleo y desempleo de la Fundación } \\
\text { Sistema Estadual de Análisis de Datos } \\
\text { (SEADE) y filtro de Hodrick-Prescott }\end{array}$ \\
\hline
\end{tabular}

Cuadro 1

Descripción de las variables utilizadas

Fuente: Elaboración propia.

11 Una fracción $\omega$ de las empresas forma expectativas basadas en el pasado, mientras que $(1-\omega)$ hace sus proyecciones con miras al futuro.

12 Los datos necesarios para la construcción de estas variables representativas para el Brasil solo están disponibles a partir de octubre de 2001. En el caso del desempleo se modificó la metodología y la nueva serie también está disponible a partir de octubre de 2001. 
El Índice Nacional de Precios al Consumidor Amplio (IPCA) es la variable representativa de la inflación ${ }^{13}$. La información proviene de la base de datos del Instituto de Investigación Económica Aplicada (Ipeadata), cuya fuente es el Sistema Nacional de Índices de Precios al Consumidor (SNIPC) del Instituto Brasileño de Geografía y Estadística (IBGE). Además de que este indicador representa la inflación considerada oficial por el Gobierno, es también el más utilizado en los estudios sobre el Brasil (Schwartzman, 2006; Areosa y Medeiros, 2007; Arruda, Ferreira y Castelar, 2011; Mendonça, Sachsida y Medrano, 2012; Sachsida, 2013).

Para considerar distintas hipótesis de expectativas con miras al futuro se utilizaron dos variables, a saber: una de previsión perfecta, bajo expectativas racionales, en la que se utiliza el IPCA adelantado, que es una forma para el modelado de las expectativas con miras al futuro (Correa y Minella, 2010; Sachsida, Ribeiro y Santos 2009), y un indicador de menor previsibilidad de los agentes, que corresponde a la media de las previsiones diarias para el mes siguiente del informe Focus del Banco Central del Brasil (Mendonça, Sachsida y Medrano, 2012; Sachsida, 2013).

La variable que incorpora el componente inercial, o basado en el pasado, de la inflación utilizada es el IPCA rezagado. Esta medida se ha empleado tradicionalmente en la mayoría de los estudios sobre el Brasil (Schwartzman, 2006; Areosa y Medeiros, 2007; Arruda, Ferreira y Castelar, 2011; Mendonça, Sachsida y Medrano, 2012; Sachsida, 2013).

En este trabajo se utilizaron tres medidas del ciclo económico: costo marginal real de las empresas, brecha del producto y brecha de desempleo. Las variables brecha del producto y brecha de desempleo se construyeron a partir de la aplicación del filtro de Hodrick-Prescott. La brecha de desempleo se incluirá como alternativa en virtud de la relación de Okun, que preconiza una relación inversa entre los ciclos del producto y del desempleo.

El costo marginal real de las empresas se construyó, conforme Galí y Gertler (1999), a partir del producto de las series de ingreso medio real efectivo de las personas ocupadas y el total de empleados - provenientes de la Encuesta Mensual de Empleo (PME) del IBGE y divulgadas por el Banco Central del Brasil - y la división del resultado entre el producto interno bruto (PIB), también proporcionado por el Banco Central del Brasil ${ }^{14}$.

En el gráfico 2 se presenta el correlograma cruzado entre algunas variables usadas en este trabajo, con miras a identificar la dirección de las interacciones entre las medidas de ciclo económico, costo marginal real de las empresas e inflación ${ }^{15}$. En el gráfico 2.A se muestra la relación entre la brecha del producto y la inflación y en el gráfico 2.B aquella entre la brecha del producto y el costo marginal. Para la composición del gráfico 2.C se sustituyó la brecha del producto por la inflación. Por último, en el gráfico 2.D se muestra la correlación entre la brecha de desempleo y la inflación.

El gráfico 2.A indica que la brecha del producto presenta una correlación positiva con la inflación futura y negativa con la inflación pasada, que justifica el hecho de que diversos autores utilizaran dicha medida rezagada en modelos empíricos de la curva de Phillips (Schwartzman, 2006; Areosa y Medeiros, 2007; Correa y Minella, 2010; Arruda, Ferreira y Castelar, 2011). Cabe resaltar que, hasta el período $t+1$ la relación negativa se mantiene - al contrario de lo que se esperaba - y corrobora el argumento de Galí y Gertler (1999) de que existen problemas para la utilización de esta variable como mecanismo para captar los efectos de los ciclos económicos en la curva de Phillips neokeynesiana.

\footnotetext{
${ }^{13}$ La población objetivo del IPCA abarca las familias con ingresos mensuales comprendidos entre 1 y 40 salarios mínimos para cualquier fuente de ingresos, incluidos los residentes en las áreas urbanas de las regiones metropolitanas.

${ }^{14}$ Los empleados se definen como aquellas personas que trabajan para un empleador o más, cumpliendo una jornada de trabajo y recibiendo en contrapartida una remuneración en dinero u otra forma de pago (vivienda, alimentación, vestuario, entre otros). Los empleados se clasifican según la existencia o no de un contrato formal de trabajo.

${ }^{15}$ El correlograma consiste en el cálculo de la correlación existente entre las variables. Las correlaciones cruzadas entre dos series, $x$ e y, están dadas por: $r_{x y}(l)=c_{x y}(l) / \sqrt{c_{x x}(0)} \cdot \sqrt{c_{y y}(0)}$, donde $l=0, \pm 1, \pm 2, \ldots$
} 
Gráfico 2

Correlograma cruzado

A. Brecha del producto $(t)$, inflación $(t+k)$

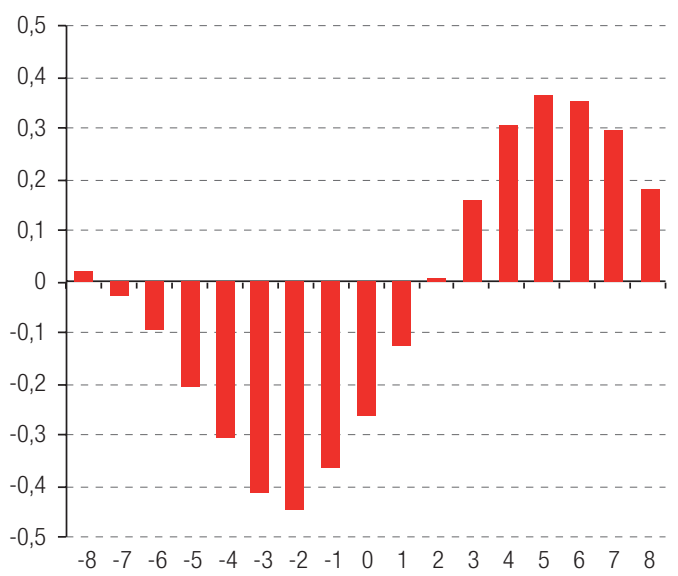

C. Costo marginal ( $t)$, inflación $(t+k)$

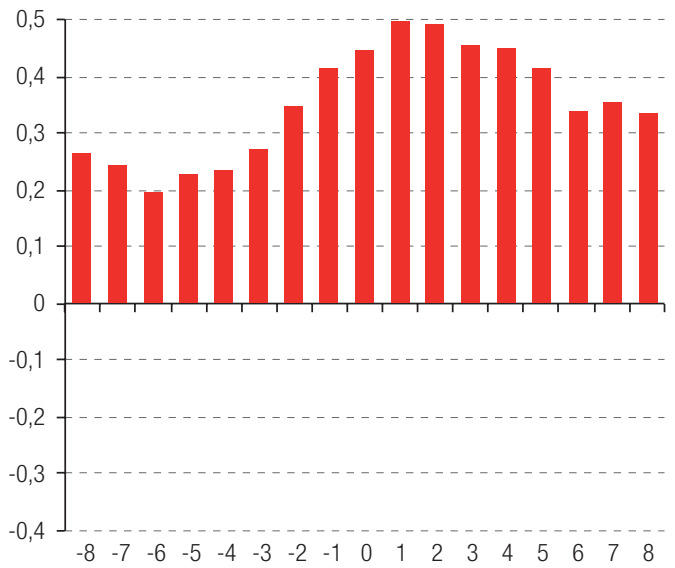

B. Brecha del producto $(t)$, costo marginal $(t+k)$

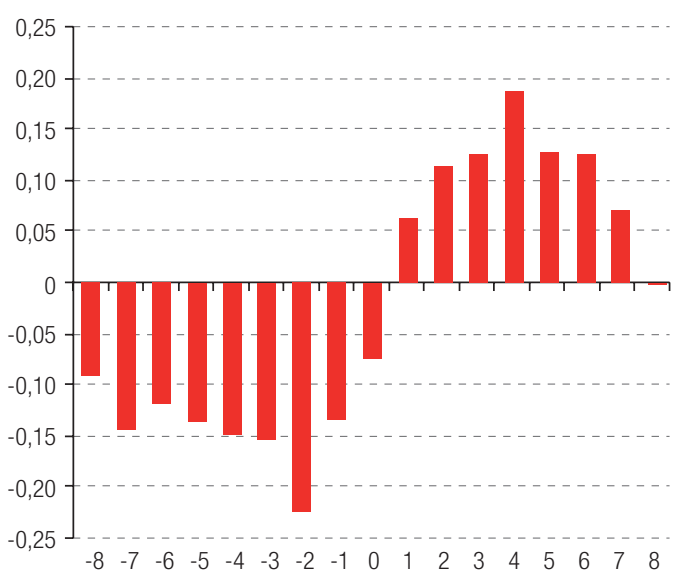

D. Brecha de desempleo $(t)$, inflación $(t+k)$

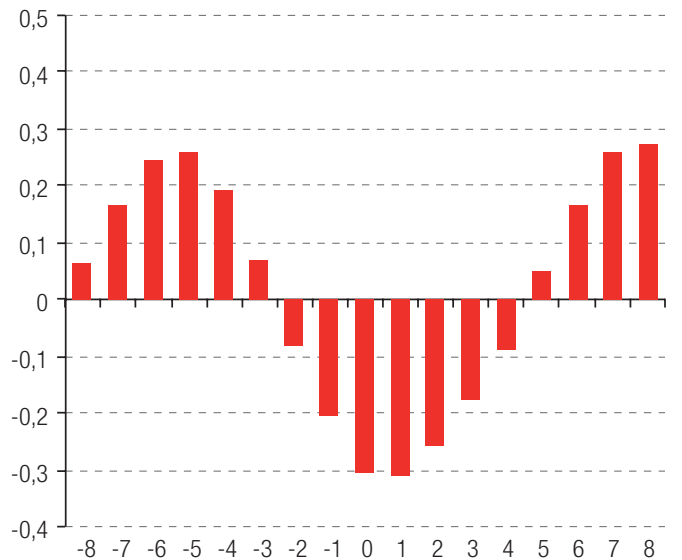

Fuente: Elaboración propia.

Para corregir ese problema, Galí y Gertler (1999) sugieren una medida de costo marginal real de las empresas. El gráfico 2.B muestra que la correlación contemporánea entre esta variable y la brecha del producto es negativa, mientras que el gráfico 2.C muestra una relación positiva entre el costo marginal real de las empresas y la inflación, que justifica el empleo de esa variable representativa. En el gráfico 2.D la medida del ciclo económico investigada es la brecha de desempleo, que presenta una correlación negativa con la inflación corriente. Es importante resaltar que, en virtud de la relación de Okun, dicha variable sigue una dirección anticíclica a la de la brecha del producto.

\section{Aspectos econométricos}

El método generalizado de momentos (MGM), propuesto por Hansen (1982), se utiliza ampliamente para la estimación de modelos de expectativas racionales pues, en esas condiciones, el método de mínimos cuadrados ordinarios (MCO) puede producir estimaciones inconsistentes, debido a que el término de error está correlacionado con algunos de los regresores endógenos. Si bien el método 
de variables instrumentales constituye una alternativa para la estimación de estos modelos, Baum, Schaffer y Stillman (2003 y 2007) muestran que, en presencia de heterocedasticidad, el MGM resulta más adecuado ${ }^{16}$.

Para seleccionar el mejor método para la estimación de los modelos aquí utilizados, inicialmente se aplica la prueba de heterocedasticidad de Pagan y Hall (1983) en la estimación de variables instrumentales ${ }^{17}$. Asimismo, se aplica la prueba de autocorrelación serial de Cumby y Huizinga (1992) ${ }^{18}$. Así, como se vio anteriormente, en caso de rechazo de la hipótesis nula de homocedasticidad en la prueba de Pagan y Hall (1983), se utiliza la estimación de MGM con corrección para este problema y, en caso de verificarse además la presencia de autocorrelación serial, se aplica un estimador de MGM consistente en presencia de heterocedasticidad y autocorrelación. Cabe resaltar que, en todos los casos analizados en este estudio, se detectó la presencia de heterocedasticidad y, por tanto, se utilizó el MGM que se describe a continuación.

Considérese la siguiente ecuación, en términos matriciales, que ha de estimarse:

$$
y=X \beta+u
$$

donde $X$ es una matriz $(n \times K)$ de regresores ${ }^{19}$ que incluye algunos regresores endógenos, o sea, $E\left(X_{i} u_{i}\right) \neq 0$ para algunas $X_{i}$. Al dividir las variables explicativas en conjuntos $\left[X_{1} X_{2}\right.$ ], con $K_{1}$ variables en $X_{1}$ consideradas endógenas y $K_{2}=\left(K-K_{1}\right)$ regresores exógenos en $X_{2}$ se obtiene:

$$
y=\left[\begin{array}{ll}
X_{1} & X_{2}
\end{array}\right]\left[\begin{array}{ll}
\beta_{1} & \beta_{2}
\end{array}\right]^{\prime}+u
$$

Sea la matriz $Z(n \times L)$ de variables instrumentales, es decir, el total de variables asumidas como exógenas, o sea, $E\left(Z_{i}, u_{i}\right)=0$. Se dividen los instrumentos como $\left[Z_{1} Z_{2}\right.$ ], en que $L_{1}$ instrumentos $Z_{1}$ se excluyen y los demás $L_{2}=\left(L-L_{1}\right)$ instrumentos $Z_{2} \equiv X_{2}$ son los instrumentos incluidos/regresores exógenos. En el diagrama 1 se sintetizan las variables.

Diagrama 1

Regresores e instrumentos

Regresores $X=\left[X_{1} X_{2}\right]=\left[X_{1} Z_{2}\right]=$ [Endógenos Exógenos $]$

Instrumentos $Z=\left[Z_{1} Z_{2}\right]$ [Excluidos Incluidos]

Fuente: Elaboración propia.

La condición para la identificación de la ecuación es que $L \geq K$, o sea, debe haber por lo menos tantos instrumentos excluidos $L_{1}$ como regresores endógenos $K_{1}$, visto que $Z_{2}$ es común a las dos listas ${ }^{20}$. Hansen (1982) muestra que, si los instrumentos son válidos, es decir, si están correlacionados con los regresores endógenos y no correlacionados con el término de error, los estimadores de MGM son consistentes y asintóticamente normales. Por tanto, se utiliza la prueba de sobreidentificación de Hansen (1982), que utiliza la hipótesis nula conjunta de que los instrumentos

\footnotetext{
${ }^{16}$ Cabe resaltar que, a pesar de ser consistente en presencia de heterocedasticidad, el estimador de MGM presenta un débil desempeño en muestras pequeñas. Por tanto, cuando el problema de heterocedasticidad no está presente, es preferible el empleo del estimador de variables instrumentales (Baum, Schaffer y Stillman, 2003 y 2007).

17 Prueba indicada para la estimación en presencia de regresores endógenos. La hipótesis nula consiste en homocedasticidad.

18 Prueba indicada para la estimación de variables instrumentales. La hipótesis nula consiste en ausencia de autocorrelación.

19 Donde $n$ es el número total de observaciones y $K$ el total de variables explicativas.

${ }^{20}$ Condición de orden. Si $L=K$, se dice que la ecuación está exactamente identificada y, si $L>K$, sobreidentificada. Cabe resaltar que la condición de orden es necesaria, pero no suficiente para la identificación.
} 
son válidos y los instrumentos $Z_{1}$ se excluyeron correctamente en el proceso de estimación ${ }^{21}$. En el caso de no rechazo de $H_{0}$, se considera que los instrumentos son válidos y que el modelo se ha estimado adecuadamente.

La suposición de que los instrumentos son exógenos puede expresarse como $E\left(Z_{i} u_{i}\right)=0$. Sea $g_{i}(\beta)$ la función $(L \times 1)$ :

$$
g_{i}(\beta)=Z_{i}^{\prime} u_{i}=Z_{i}^{\prime}\left(y_{i}-X_{i} \beta\right)
$$

Así, la exogeneidad de los instrumentos requiere que existan $L$ condiciones de momento, o de ortogonalidad, de manera que $E\left(g_{i}(\beta)\right)=0$. La contrapartida muestral de esa condición de momento corresponde a:

$$
\bar{g}(\hat{\beta})=\frac{1}{n} \sum_{i=1}^{n} g_{i}(\hat{\beta})=\frac{1}{n} \sum_{i=1}^{n} Z_{i}^{\prime}\left(y_{i}-X_{i} \hat{\beta}\right)=\frac{1}{n} Z^{\prime} \hat{u}
$$

La intuición del método es elegir un estimador para $\beta$ que minimice $\bar{g}(\hat{\beta})$ preferiblemente hasta igualarla a 0 . Por tanto, el estimador MGM para $\beta$ es la $\hat{\beta}$ que minimiza la función objetivo $J(\hat{\beta})$ :

$$
\hat{\beta}_{G M M}=\arg \min J(\hat{\beta})=n \bar{g}(\hat{\beta})^{\prime} W \bar{g}(\hat{\beta})
$$

en que $W$ es una matriz de pesos $(L \times L)$ utilizada en la construcción de una forma cuadrática para las condiciones de momento. El estimador eficiente de MGM emplea una matriz de pesos óptima $W=S^{-1}$ que minimiza la varianza asintótica del estimador. Al derivar las condiciones de primer orden del problema anterior se obtiene:

$$
\hat{\beta}_{G M M}=\left(X^{\prime} Z W Z^{\prime} X\right)^{-1}\left(X^{\prime} Z W Z^{\prime} y\right)
$$

Por tanto, para la estimación MGM de este ejercicio empírico, los instrumentos incluyen hasta seis rezagos de la inflación, la brecha del producto, el costo marginal de las empresas, la brecha de desempleo y la tasa de interés, conforme Galí y Gertler (1999). Las condiciones de ortogonalidad de la curva de Phillips neokeynesiana y de su versión híbrida están representadas, respectivamente, por las ecuaciones (18) y (19):

$$
\begin{gathered}
E_{t}\left\{\left(\pi_{t}-\lambda m c_{t}-\gamma_{f} \pi_{t+1}\right) z_{t}\right\}=0 \\
E_{t}\left\{\left(\pi_{t}-\lambda m c_{t}-\gamma_{f} \pi_{t+1}-\gamma_{b} \pi_{t-1}\right) z_{t}\right\}=0
\end{gathered}
$$

En síntesis, la estrategia econométrica para la estimación de los modelos utilizados en este estudio consiste inicialmente en la investigación de la presencia de heterocedasticidad y autocorrelación serial en la estimación de variables instrumentales mediante las pruebas de Pagan y Hall (1983) y Cumby y Huizinga (1992), respectivamente ${ }^{22}$. Al rechazarse la $H_{0}$ de homocedasticidad, se utiliza el MGM con corrección para este problema. En caso de verificarse también la presencia de autocorrelación serial, se aplica la corrección para los dos problemas, es decir, el MGM consistente en presencia de heterocedasticidad y autocorrelación. Por último, se realiza la prueba de Hansen (1982) de validez de los instrumentos y, en caso de que no se rechace $H_{0}$, se considera que los instrumentos son válidos y que, por tanto, el modelo se ha estimado adecuadamente.

${ }^{21}$ El estimador de MGM utiliza la estadística J, de Hansen (1982), que sigue una $X_{L-K}^{2}$. En la estimación por variables instrumentales $(\mathrm{VI})$, la estadística utilizada es $n R^{2}$, extraída de una regresión auxiliar de los residuos $\mathrm{VI}$ sobre el conjunto completo de los instrumentos, y también sigue una $X_{L-K}^{2}$.

22 Si los errores son homocedásticos, Baum, Schaffer y Stillman (2003 y 2007) muestran que es preferible el empleo del estimador de variables instrumentales, en virtud del débil desempeño del MGM en muestras pequeñas. 


\section{Análisis y discusión de los resultados}

Para examinar el comportamiento dinámico de la inflación brasileña considerando distintas hipótesis de expectativas en la curva de Phillips neokeynesiana y su versión híbrida se utilizaron el MGM consistente en presencia de heterocedasticidad y autocorrelación y datos mensuales del período comprendido entre enero de 2002 y diciembre de 2012.

De esta forma, se obtienen dos situaciones de expectativas relacionadas con la previsibilidad de los agentes económicos, a saber: previsión perfecta —en la que se utiliza el IPCA adelantado como expectativas con miras al futuro - y menor previsibilidad - en la que se utiliza la media de las expectativas de inflación del informe Focus del Banco Central del Brasil. Además, en cada modelo se consideran tres variables distintas para los ciclos económicos: el costo marginal real de las empresas, la brecha del producto y la brecha de desempleo. Cabe resaltar que, inicialmente, todas las variables se sometieron a las pruebas de raíz unitaria de Dickey-Fuller aumentada y de Phillips-Perron y resultaron estacionarias a los niveles usuales de significación. Los resultados se sintetizan en el cuadro 2.

Cuadro 2

Pruebas de raíz unitaria

\begin{tabular}{lll}
\hline \multirow{2}{*}{ Serie } & \multicolumn{1}{c}{$\begin{array}{c}\text { Dickey-Fuller aumentada } \\
\text { Estadística de prueba en nivel } \\
\text { Nivel } \\
\text { (valor p) }\end{array}$} & $\begin{array}{c}\text { Phillips-Perron } \\
\text { Estadística de prueba } \\
\text { Nivel } \\
\text { (valor p) }\end{array}$ \\
\hline $\begin{array}{l}\text { Inflación (Índice Nacional de Precios } \\
\text { al Consumidor Amplio (IPCA)) }\end{array}$ & $-4,6799$ & $-4,3583$ \\
\hline Costo marginal & $(0,0013)$ & $(0,0006)$ \\
\hline Previsión Focus & $-5,7642$ & $-5,6681$ \\
\hline Brecha de desempleo & $(0,0000)$ & $(0,0000)$ \\
\hline Brecha del producto & $-4,3523$ & $-6,3667$ \\
\hline
\end{tabular}

Fuente: Elaboración propia.

Nota: Valor p entre paréntesis.

Así, se obtienen 6 modelos para cada hipótesis de expectativas con miras al futuro, que totalizan 12 modelos estimados ${ }^{23}$. En los cuadros 3 y 4 se sintetizan los resultados de las estimaciones de la curva de Phillips neokeynesiana y de su versión híbrida, respectivamente.

La prueba de Pagan y Hall (1983) indicó la presencia de heterocedasticidad en todos los modelos en la estimación por variables instrumentales y, en consecuencia, se optó por el uso del MGM. La ausencia de autocorrelación según la prueba de Cumby y Huizinga (1992) solo se verificó en los modelos de la curva de Phillips neokeynesiana con previsión perfecta y brecha del producto como variable del ciclo y en los modelos de la curva de Phillips neokeynesiana híbrida bajo incertidumbre (previsión Focus) y costo marginal real de las empresas como variable del ciclo. En esos casos, el MGM se corrigió solo para heterocedasticidad. Asimismo, en ninguno de los modelos estimados se rechaza la $H_{0}$ de validez de los instrumentos por la prueba de Hansen (1982), de manera que, en estas condiciones, los modelos se han estimado adecuadamente.

\footnotetext{
${ }^{23}$ Es decir, seis modelos para la curva de Phillips neokeynesiana y seis para la curva de Phillips neokeynesiana híbrida.
} 


\section{Cuadro 3}

Estimación de la curva de Phillips neokeynesiana con el método generalizado de momentos

(MGM) consistente en presencia de heterocedasticidad y autocorrelación

\begin{tabular}{|c|c|c|c|c|c|c|}
\hline \multirow{2}{*}{ Expectativas } & \multirow{2}{*}{ Ciclos económicos } & \multicolumn{2}{|c|}{ Parámetros } & \multirow{2}{*}{$\begin{array}{l}\text { Prueba J } \\
\text { Hansen }\end{array}$} & \multirow{2}{*}{$\begin{array}{c}\text { Heterocedasticidad } \\
\text { Pagan y Hall }\end{array}$} & \multirow{2}{*}{$\begin{array}{l}\text { Autocorrelación } \\
\text { Cumby y Huizinga }\end{array}$} \\
\hline & & $\lambda$ & $\gamma_{f}$ & & & \\
\hline \multirow{6}{*}{$\begin{array}{l}\text { Previsión } \\
\text { perfecta }\end{array}$} & \multirow{2}{*}{ Costo marginal } & 0,30 & 0,39 & 4,03 & $X^{2}(5)=29,12$ & $X^{2}(1)=6,92$ \\
\hline & & $(0,01)$ & $(0,00)$ & $(0,25)$ & $(0,00)$ & $(0,00)$ \\
\hline & \multirow{2}{*}{$\begin{array}{l}\text { Brecha de } \\
\text { desempleo }\end{array}$} & $-0,41$ & 0,34 & 7,97 & $X^{2}(14)=21,14$ & $X^{2}(1)=12,10$ \\
\hline & & $(0,04)$ & $(0,01)$ & $(0,78)$ & $(0,04)$ & $(0,00)$ \\
\hline & \multirow{2}{*}{$\begin{array}{l}\text { Brecha del } \\
\text { producto }^{\mathrm{a}}\end{array}$} & $-1,97$ & 0,59 & 4,43 & $X^{2}(5)=15,54$ & $X^{2}(1)=0,25 a$ \\
\hline & & $(0,00)$ & $(0,01)$ & $(0,22)$ & $(0,00)$ & $(0,62)$ \\
\hline \multirow{6}{*}{ Previsión Focus } & \multirow{2}{*}{ Costo marginal } & 0,38 & 1,09 & 3,23 & $X^{2}(5)=20,19$ & $X^{2}(1)=43,13$ \\
\hline & & $(0,00)$ & $(0,00)$ & $(0,35)$ & $(0,00)$ & $(0,00)$ \\
\hline & \multirow{2}{*}{$\begin{array}{l}\text { Brecha de } \\
\text { desempleo }\end{array}$} & $-0,91$ & 1,23 & 7,82 & $X^{2}(15)=27,13$ & $X^{2}(1)=43,13$ \\
\hline & & $(0,00)$ & $(0,00)$ & $(0,85)$ & $(0,02)$ & $(0,00)$ \\
\hline & \multirow{2}{*}{$\begin{array}{l}\text { Brecha del } \\
\text { producto }\end{array}$} & $-0,24$ & 1,27 & 5,87 & $X^{2}(15)=23,55$ & $X^{2}(15)=46,94$ \\
\hline & & $(0,52)$ & $(0,00)$ & $(0,95)$ & $(0,05)$ & $(0,00)$ \\
\hline
\end{tabular}

Fuente: Elaboración propia sobre la base de la ecuación $\pi_{t}=\lambda x_{t}+\gamma_{f} E_{t}\left\{\pi_{t+1}\right\}$.

Nota: Valor $\mathrm{p}$ entre paréntesis. Las pruebas de autocorrelación y heterocedasticidad se aplicaron en la estimación con variables instrumentales.

a Modelo con corrección solo para la heterocedasticidad.

\section{Cuadro 4}

Estimación de la curva de Phillips neokeynesiana híbrida con el método generalizado de momentos (MGM) consistente en presencia de heterocedasticidad y autocorrelación

\begin{tabular}{|c|c|c|c|c|c|c|c|}
\hline \multirow{2}{*}{ Expectativas } & \multirow{2}{*}{$\begin{array}{l}\text { Ciclos } \\
\text { económicos }\end{array}$} & \multicolumn{3}{|c|}{ Parámetros } & \multirow{2}{*}{$\begin{array}{l}\text { Prueba J } \\
\text { Hansen }\end{array}$} & \multirow{2}{*}{$\begin{array}{c}\text { Heterocedasticidad } \\
\text { Pagan y Hall }\end{array}$} & \multirow{2}{*}{$\begin{array}{l}\text { Autocorrelación } \\
\text { Cumby y Huizinga }\end{array}$} \\
\hline & & $\lambda$ & $\gamma_{f}$ & $\gamma_{b}$ & & & \\
\hline \multirow{6}{*}{$\begin{array}{l}\text { Previsión } \\
\text { perfecta }\end{array}$} & \multirow{2}{*}{ Costo marginal } & 0,17 & 0,30 & 0,53 & 5,37 & $X^{2}(10)=22,35$ & $X^{2}(1)=15,69$ \\
\hline & & $(0,01)$ & $(0,00)$ & $(0,00)$ & $(0,61)$ & $(0,01)$ & $(0,00)$ \\
\hline & \multirow{2}{*}{$\begin{array}{l}\text { Brecha de } \\
\text { desempleo }\end{array}$} & $-0,26$ & 0,39 & 0,52 & 8,77 & $X^{2}(16)=25,98$ & $X^{2}(1)=32,75$ \\
\hline & & $(0,01)$ & $(0,00)$ & $(0,00)$ & $(0,79)$ & $(0,05)$ & $(0,00)$ \\
\hline & \multirow{2}{*}{$\begin{array}{l}\text { Brecha del } \\
\text { producto }\end{array}$} & $-0,30$ & 0,30 & 0,55 & 6,35 & $X^{2}(16)=25,50$ & $X^{2}(1)=33,15$ \\
\hline & & $(0,23)$ & $(0,03)$ & $(0,00)$ & $(0,49)$ & $(0,06)$ & $(0,00)$ \\
\hline \multirow{6}{*}{$\begin{array}{l}\text { Previsión } \\
\text { Focus }\end{array}$} & \multirow{2}{*}{$\begin{array}{l}\text { Costo } \\
\text { marginala }\end{array}$} & 0,31 & 0,22 & 0,61 & 9,07 & $X^{2}(17)=38,83$ & $X^{2}(1)=0,99^{*}$ \\
\hline & & $(0,00)$ & $(0,03)$ & $(0,00)$ & $(0,82)$ & $(0,00)$ & $(0,32)$ \\
\hline & \multirow{2}{*}{$\begin{array}{l}\text { Brecha de } \\
\text { desempleo }\end{array}$} & $-0,75$ & 0,24 & 0,63 & 6,94 & $X^{2}(14)=28,69$ & $X^{2}(1)=16,22$ \\
\hline & & $(0,00)$ & $(0,00)$ & $(0,00)$ & $(0,80)$ & $(0,01)$ & $(0,00)$ \\
\hline & \multirow{2}{*}{$\begin{array}{l}\text { Brecha del } \\
\text { producto }\end{array}$} & 0,16 & 0,22 & 0,67 & 7,34 & $X^{2}(16)=35,23$ & $X^{2}(1)=11,24$ \\
\hline & & $(0,48)$ & $(0,03)$ & $(0,00)$ & $(0,77)$ & $(0,01)$ & $(0,00)$ \\
\hline
\end{tabular}

Fuente: Elaboración propia sobre la base de la ecuación $\pi_{t}=\lambda x_{t}+\gamma_{f} E_{t}\left\{\pi_{t+1}\right\}+\gamma_{b} \pi_{t-1}$.

Nota: Valor $p$ entre paréntesis. Las pruebas de autocorrelación y heterocedasticidad se aplicaron en la estimación con variables instrumentales.

a Modelo con corrección solo para la heterocedasticidad.

La variable brecha del producto resulta estadísticamente igual a 0 o presenta un signo contrario al previsto en la teoría. Este resultado confirma la evidencia destacada en la literatura nacional (Mazali y Divino, 2010; Mendonça, Sachsiday Medrano, 2012; Sachsida, 2013) einternacional (Galíy Gertler, 1999; Galí, Gertler y López-Salido, 2001), que corrobora la dificultad que supone el empleo de ese indicador, como medida del ciclo económico, para explicar la dinámica de la inflación.

A pesar de ello, se puede decir que, para el período analizado, la curva de Phillips neokeynesiana y su versión híbrida constituyeron mecanismos robustos para explicar la dinámica inflacionaria brasileña, pues las variables de los ciclos económicos - medidas por el costo marginal real de las empresas y por la brecha de desempleo - resultaron estadísticamente relevantes y presentaron los signos 
previstos en la teoría. La evidencia también indica la significación estadística de los componentes de expectativas basadas en el pasado y con miras al futuro y que el impacto del primero es mayor que el del segundo. Esto sugiere que la dinámica reciente de la inflación brasileña todavía presenta un marcado componente inercial. En otras palabras, conforme Simonsen (1970 y 1985), Cabello (2014) y Carvalho (2014), la memoria de hiperinflaciones y el aumento reciente de la indexación en la economía brasileña pueden explicar esa mayor influencia de la inercia inflacionaria.

Los resultados parecen indicar además que, en una hipótesis de menor previsibilidad de los agentes económicos, la inflación es más vulnerable a las oscilaciones cíclicas de la actividad económica, pues el impacto de esas variables es mucho mayor, en valores absolutos, cuando se considera la previsión Focus como variable de expectativas con miras al futuro. Por tanto, como destacan Sicsú (2002) y Mendonça (2002 y 2004), cuanto menor sea la capacidad predictiva de los agentes económicos, mayor será el costo de una política de desinflación en términos de oscilaciones cíclicas en la actividad económica.

Por último, la evidencia también sugiere que, en un marco de menor previsibilidad, la inflación es más susceptible al componente inercial, o basado en el pasado, pues la repercusión de esa variable en la dinámica inflacionaria resulta superior en todos los modelos considerados.

\section{VII.Consideraciones finales}

En el presente trabajo se analizó la dinámica de la inflación brasileña considerando distintas hipótesis de expectativas en la curva de Phillips neokeynesiana y la curva de Phillips neokeynesiana híbrida, utilizando datos mensuales del período comprendido entre enero de 2002 y diciembre de 2012 y el MGM consistente en presencia de heterocedasticidad y autocorrelación.

En términos generales, los resultados confirmaron la inadecuación de la brecha del producto y la robustez de la brecha de desempleo y del costo marginal de las empresas como mecanismos para captar el efecto de transmisión de los ciclos económicos a la inflación del Brasil en el período analizado. En otras palabras, la medida de costo marginal real de las empresas empleada en este estudio constituye una alternativa para aplicaciones futuras en el Brasil. Además, los resultados sugieren la relevancia de las expectativas basadas en el pasado y con miras al futuro y que el impacto de las primeras es superior, de manera que la dinámica inflacionaria brasileña todavía presenta un importante componente inercial. Así, puede decirse que la curva de Phillips neokeynesiana y su versión híbrida son marcos adecuados para la investigación de la dinámica reciente de la inflación brasileña.

La evidencia sugiere, además, que la inflación es más sensible a las oscilaciones en los ciclos económicos cuanto menor sea la previsibilidad de los agentes económicos. De hecho, como argumentan Sicsú (2002) y Mendonça (2002 y 2004), cuanto menor sea la capacidad de los agentes de prever el comportamiento de la inflación, mayor será el costo de una política de desinflación en términos de actividad económica.

Por último, el componente inercial de la inflación parece tener un efecto mayor cuando se considera una hipótesis de menor previsibilidad. O sea, el aumento reciente del grado de indexación de la economía brasileña relacionado con su memoria de hiperinflación parece influir en el mecanismo de la inercia inflacionaria y, además, la menor previsibilidad supone un gran costo en términos de reproducción de la inflación pasada en el presente.

En resumen, a partir de los resultados obtenidos se puede concluir que, cuanto mayor sea la previsibilidad de los agentes económicos, menos onerosas serán las políticas de desinflación, tanto en términos de oscilaciones cíclicas de la actividad económica como de transmisión inercial de la inflación. 


\section{Bibliografía}

Areosa, W. D. y M. Medeiros (2007), "Inflation dynamics in Brazil: the case of a small open economy", Brazilian Review of Econometrics, vol. 27, № 1, Río de Janeiro.

Arruda, E. F., R. T. Ferreira e I. Castelar (2011), "Modelos lineares e não lineares da curva de Phillips para a previsão da taxa de inflação no Brasil”, Revista Brasileira de Economia, vol. 65, № 3, Río de Janeiro, Fundación Getulio Vargas.

Baum, C. F., M. E. Schaffer y S. Stillman (2007), "Enhanced routines for instrumental variables/generalized method of moments estimation and testing", The Stata Journal, vol. 7, № 4. (2003), "Instrumental variables and GMM: estimation and testing", The Stata Journal, vol. 3, № 1.

Bresser-Pereira, L. C. y C. Gomes da Silva (2008), "Inflation targeting in Brazil: a Keynesian approach", Keynes and Macroeconomics after 70 Years: Critical Assessments of the General Theory, L. R. Wray y M. Forstater (eds.), Cheltenham, Edward Elgar.

Cabello, A. F. (2014), "Mário Henrique Simonsen e a construção do conceito de inflação inercial", Revista de Economia Política, vol. 34, № 2, São Paulo, Centro de Economía Política.

Calvo, G. A. (1983), "Staggered prices in a utility-maximizing framework", Journal of Monetary Economics, vol. 12, N ${ }^{\circ}$ 3, Amsterdam, Elsevier.

Carvalho, A. R. (2014), "A persistência da indexação no Brasil pós-real", Revista de Economia Política, vol. 34, № 2, São Paulo, Centro de Economía Política.

Correa, A. S. y A. Minella (2010), "Nonlinear mechanisms of the exchange rate pass-through: a Phillips curve model with threshold for Brazil”, Revista Brasileira de Economia, vol. 64, № 3, Río de Janeiro, Fundación Getulio Vargas.

Cumby, R. E. y J. Huizinga (1992), "Testing the autocorrelation structure of disturbances in ordinary least squares and instrumental variables regressions", Econometrica, vol. 60, № 1, Nueva York, The Econometric Society.

Friedman, M. (1977), "Nobel lecture: inflation and unemployment", Journal of Political Economy, vol. 85, № 3, Chicago, The University of Chicago Press.

(1968), "The role of monetary policy", American Economic Review, vol. 58, № 1, Nashville, Tennessee, American Economic Association.

Galí, J. y M. Gertler (1999), "Inflation dynamics: a structural econometric analysis", Journal of Monetary Economics, vol. 44, № 2, Amsterdam, Elsevier.

Galí, J., M. Gertler y J. D. López-Salido (2001), "European inflation dynamics", European Economic Review, vol. 45, № 7, Amsterdam, Elsevier.

Giambiagi, F. y otros (2011), Economia brasileira contemporânea, Río de Janeiro, Elsevier Editora Ltda.

Hansen, L. P. (1982), "Large sample properties of generalized method of moments estimators", Econometrica, vol. 50, № 4, Nueva York, The Econometric Society.

Lucas, R. E. (1972), "Expectations and the neutrality of money", Journal of Economic Theory, vol. 4, № 2, Amsterdam, Elsevier.

Mazali, A. A. y J. A. Divino (2010), "Real wage rigidity and the New Phillips curve: the Brazilian case", Revista Brasileira de Economia, vol. 64, №3, Río de Janeiro, Fundación Getulio Vargas.

Mendonça, H. F. (2004), "Mensurando a credibilidade do regime de metas inflacionárias no Brasil", Revista de Economia Política, vol. 24, № 3, São Paulo, Centro de Economía Política. (2002), "A teoria da credibilidade da política monetária", Revista de Economia Política, vol. 22, № 3, São Paulo, Centro de Economía Política.

Mendonça, H. F. y M. A. L. dos Santos (2006), "Credibilidade da política monetária e a previsão do trade-off entre inflação e desemprego: uma aplicação para o Brasil”, Economia, vol. 7, № 2, Brasilia, Asociación Nacional de Centros de Posgrado en Economía.

Mendonça, M. J. C., A. Sachsida y L. Medrano (2012), "Inflação versus desemprego: novas evidências para o Brasil", Economia Aplicada, vol. 16, № 3, São Paulo, Universidad de São Paulo.

Mesquita, M. (2014), "A política econômica do governo Dilma: a volta do experimentalismo", Sob a luz do sol: uma agenda para o Brasil, São Paulo, Centro de Debate de Políticas Públicas (CDPP).

Muth, J. F. (1961), "Rational expectations and the theory of price movements", Econometrica, vol. 29, № 3, Nueva York, The Econometric Society.

Pagan, A. R. y D. Hall (1983), "Diagnostic tests as residual analysis", Econometric Reviews, vol. 2, № 2, Taylor \& Francis. 
Phelps, E. S. (1969), "The new microeconomics in inflation and employment theory", American Economic Review, vol. 59, Nㅜㄹ. Nashville, Tennessee, American Economic Association.

_ (1967), "Phillips curves, expectations of inflation and optimal unemployment over time", Economica, vol. 34, N 135, Wiley.

Phillips, A. W. (1958), "The relationship between unemployment and the rate of change of money wage rates in the United Kingdom, 1861-1957", Economica, vol. 25, № 100, Wiley.

Rudd, J. y K. Whelan (2005), "New tests of the new-Keynesian Phillips curve", Journal of Monetary Economics, vol. 52, N 6, Amsterdam, Elsevier.

Sachsida, A. (2013), "Inflação, desemprego e choques cambiais: uma revisão da literatura sobre a curva de Phillips no Brasil”, Revista Brasileira de Economia, vol. 67, Nㅜ 4, Río de Janeiro, Fundación Getulio Vargas.

Sachsida, A., M. Ribeiro y C. H. dos Santos (2009), "A curva de Phillips e a experiência brasileira", Texto para Discussão, № 1459, Brasilia, Instituto de Investigación Económica Aplicada (IPEA).

Sargent, T. J. (1971), "A note on the 'accelerationist' controversy", Journal of Money, Credit and Banking, vol. 3, No 3, Ohio, Ohio State University Press.

Schwartzman, F. (2006), "Estimativa de curva de Phillips para o Brasil com preços desagregados", Economia Aplicada, vol. 10, № 1, São Paulo, Universidad de São Paulo.

Sicsú, J. (2002), "Expectativas inflacionárias no regime de metas de inflação: uma análise preliminar do caso brasileiro", Economia Aplicada, vol. 6, № 4, São Paulo, Universidad de São Paulo.

Simonsen, M. H. (1970), Inflação: gradualismo x tratamento de choque, Río de Janeiro, APEC Editora.

_ (1985), "Contratos salariais justapostos e política anti-inflacionária", Revista de Econometria, vol. 5, № 2, Río de Janeiro.

Taylor, J. B. (1980), "Aggregate dynamics and staggered contracts", Journal of Political Economy, vol. 88, $N^{\circ} 1$, Chicago, The University of Chicago Press. 